\title{
Radiotherapy for early glottic cancer and salvage surgery after recurrence
}

\author{
Paulo Pontes $^{1}$, Osíris de Oliveira Camponês do Brasil ${ }^{2}$, Francisco de Souza Amorim Filho ${ }^{3}$, Bruno Teixeira \\ de Moraes $^{4}$, Antonio Pontes 5 , José Caporrino Neto ${ }^{6}$
}

\section{Keywords:}

laryngeal neoplasms, laryngectomy, neoplasm recurrence local,

radiotherapy, salvage therapy.

\begin{abstract}
$\mathbf{E}$ arly glottic cancer can be effectively treated with radiation or surgery, but recurrence is a possibility when using any of the treatment modalities.
\end{abstract}

\begin{abstract}
Aim: To assess the outcome of radiotherapy as initial treatment in the control of squamous cell carcinoma of vocal cord (T1) and the effectiveness of salvage surgery (endoscopic or open) after treatment failure.
\end{abstract}

Materials and Methods: A retrospective study was based on the analysis of medical records from 43 patients with T1 squamous cell carcinoma of the glottis, radiotherapy as initial treatment and follow-up period of five years.

Results: The rate of recurrence after radiotherapy was 30.2\% of the cases, mean diagnosis interval was 29.5 months. As an option for salvage treatment, patients underwent open partial laryngectomy or endoscopic surgery with control rates of $77.7 \%$ and $25 \%$ respectively.

Conclusion: Our cases showed high rates of recurrence after radiotherapy and open partial laryngectomy was more effective for the salvage surgery.

${ }^{1}$ Full Professor - Department of Otorhinolaryngology and Head and Neck Surgery of the Federal University of São Paulo, UNIFESP-EPM. ${ }^{2}$ PhD in Sciences; Professor - Department of Otorhinolaryngology and Head and Neck Surgery of the Federal University of São Paulo, UNIFESP-EPM. ${ }^{3}$ Head and Neck Surgeon; PhD in Sciences - Department of Otorhinolaryngology and Head and Neck Surgery of the Federal University of São Paulo, UNIFESP-EPM ${ }^{4}$ Otorhinolaryngologist; MSc student - Department of Otorhinolaryngology and Head and Neck Surgery of the Federal University of São Paulo, UNIFESP-EPM.

${ }^{5}$ Otorhinolaryngologist; MSc student - Department of Otorhinolaryngology and Head and Neck Surgery of the Federal University of São Paulo, UNIFESP-EPM.

${ }^{6} \mathrm{PhD}$ in Sciences; Assistant Physician - Department of Otorhinolaryngology and Head and Neck Surgery of the Federal University of São Paulo, UNIFESP-EPM. Institute of the Larynx (INLAR).

Send correspondence to: Paulo Pontes - Rua Diogo de Faria 231 Vila Clementino São Paulo SP 04037-000.

Tel. (+55 11) 5549-2188 - Fax: (011) 5549-2127 - E-mail: ppontes@inlar.com.br

Paper submitted to the BJORL-SGP (Publishing Management System - Brazilian Journal of Otorhinolaryngology) on January 22, 2010; and accepted on December 8, 2010. cod. 6890 


\section{INTRODUCTION}

Malignant laryngeal neoplasm is one of the most frequent diseases of the head and neck, and in 90\% of the cases it is represented by the epidermoid carcinoma and its genesis is directly associated with alcohol drinking and smoking.

It more frequently affects the glottis - $64 \%$ of the cases, and any epithelial disarray or change caused by the tumor will change the vibratory movements of the vocal folds, causing dysphonia. Of all the glottis tumors, 56\% are diagnosed in their initial stages (T1) ${ }^{1}$.

The primary glottis cancer can be effectively treated with radiotherapy or surgery (endoscopic or open) and it bears some of the best cure rates among the malignant neoplasms of the aerodigestive tract. In the series published by Davis et al. ${ }^{2}$, they reported a local control rate of $93 \%$ in patients with glottic T1 tumors submitted to surgery and 67\% in T1 patients submitted to radiotherapy. Nonetheless, when we consider only the T1a stage tumors, some authors report similar control rates with cordectomy and radiotherapy, $84 \%$ and $77 \%$ respectively. ${ }^{3}$

Treatment must not be restricted only to curing the cancer, which is the main objective, but one must also attempt to preserve breathing, swallowing and phonation.

\section{OBJECTIVE}

To assess the results of radiotherapy as the initial treatment mode in controlling the epidermoid carcinoma of the vocal fold (T1) and the efficacy of surgical salvage (endoscopic or open) after initial treatment failure.

\section{MATERIALS AND METHODS}

The present study was approved by the Ethics in Research Committee of the institution to which this study is associated, under registration number 0353/10.

In this series we retrospectively assessed the charts from 43 patients with T1 stage glottis epidermoid carcinoma (42 men and 1 woman), submitted to radiotherapy as initial treatment, who were first seen by a doctor between 1990 and 2002.

All the patients were sorted according to the TNMUICC 2002 classification, after indirect laryngoscopy obtained by tele-laryngoscopy and/or nasal fibroscopic laryngoscopy (saved in video) and diagnostic confirmation of the epidermoid carcinoma through biopsy/frozen section.

We took off the sample those patients who had been previously treated in other services, those with tumors bearing other clinical stages and those with tumors of histological lineage other than the epidermoid carcinoma.

The routine in our service is to present all treatment options (radiotherapy or surgery) to patients with T1 glottic carcinoma, explaining the respective pros and cons; nonetheless, surgery is our treatment of choice for these cases. Radiation treatment was based on external beam at the dose of 6,000 to 6,800 rad in 30-34 fractions, indicated according to two basic criteria:

$28 \%)$

- Patient clinically ineligible for surgery $(n=12 /$

- Patient's wish ( $\mathrm{n}=31 / 72 \%)$

In order to analyze the results obtained by radiotherapy, the patients were grouped according to primary tumor localization on the vocal folds as follows:

- Group A - T1a: Tumor not involving the anterior commissure $(n=22)$.

- Group B - T1b: Tumor involving the anterior commissure with or without invasion of the contralateral fold anterior third $(n=21)$.

After radiotherapy, all the patients were followed up for a period of five years or more, and in the first six months they returned monthly to the clinic. For clinical control we did indirect laryngoscopy with the goal of identifying any suspicious lesions early on. After tumor recurrence diagnosis, the situation was recorded in the patient's chart and the patient was referred to salvage treatment as soon as possible, which first option was partial open laryngectomy (frontolateral vertical) in the cases with or without commissure involvement. Patients with T1a tumors upon recurrence who were clinically unfit were submitted to an endoscopic tumor resection (transmuscular cordectomy), since it is an approach with less morbidity than open surgery.

\section{RESULTS}

The patients submitted to radiotherapy $(\mathrm{N}=43)$ to treat initial glottic cancer had local recurrence in 30.2\% $(\mathrm{N}=13)$ of the cases. Everyone who had recurrences was male with an average age of 58.9 years. The mean time between radiotherapy onset and local recurrence diagnosis was 29.5 months. The most frequent histology type was the moderately differentiated epidermoid carcinoma (76.9\%), followed by the well differentiated type (23.1\%).

As to primary tumor location, initial radiotherapy treatment had the following recurrences: In Group A, where the initial lesion did not involve the anterior commissure, there was recurrence in $31.8 \%(\mathrm{n}=7)$ of the cases (Table 1); in Group B, in which the initial lesion invaded the anterior commissure with or without involvement of the contralateral vocal fold, the recurrence rate was $28.5 \%$ $(n=6)$, slightly lower than that of the previous group, when radiotherapy was carried out (Table 2).

As soon as the failure in radiotherapy was confirmed, the patients were once again staged, being similar to the staging before radiotherapy with no loco regional metastasis. Four patients were referred to salvage endoscopic surgery (transmuscular cordectomy), because they were clinically unfit. Nine patients were submitted to open 
surgery (vertical-frontolateral laryngectomy) without neck dissection (Table 3).

Table 1. Group A (T1a): treatment by radiotherapy versus tumor recurrence.

\begin{tabular}{lll}
\hline Recurrence & \multicolumn{2}{l}{ Radiotherapy } \\
\hline & $\mathrm{N}$ & $\%$ \\
With recurrence & 7 & 31,8 \\
Without recurrence & 15 & 68,2 \\
Total & 22 & 100 \\
\hline
\end{tabular}

Table 2. Group B (T1b): treatment by radiotherapy versus tumor recurrence.

\begin{tabular}{lll}
\hline Recurrence & \multicolumn{2}{c}{ Radiotherapy } \\
\hline & $\mathrm{N}$ & $\%$ \\
With recurrence & 6 & 28,5 \\
Without recurrence & 15 & 71,5 \\
Total & 21 & 100 \\
\hline
\end{tabular}

Table 3. Local recurrence according to treatment.

\begin{tabular}{cccc}
\hline Recurrence & \multicolumn{2}{c}{ Treatment } & Total \\
\hline & Open surgery & $\begin{array}{l}\text { Endoscopic } \\
\text { procedure }\end{array}$ \\
Cured & $77,7 \%(n=7)$ & $25 \%(n=1)$ & $69,20 \%$ \\
Uncured & $22,2 \%(n=2)$ & $75 \%(n=3)$ & $30,80 \%$ \\
Total & $N=9$ & $N=4$ & $N=13$ \\
\hline
\end{tabular}

Of the recurrence cases treated endoscopically $(n=4)$, only one was cured, the remaining patients $(n=3)$ were referred to total laryngectomy because of a new recurrence. In this group, one patient died of cancer.

In the cases submitted to open surgery, the cure rate was higher, and $77.7 \%(\mathrm{n}=7)$ patients benefited from surgery. $22.2 \%(n=2)$ of the patients had new treatment failure and were referred to total laryngectomy. In this group we also had one patient dying of cancer.

\section{DISCUSSION}

The treatment of laryngeal cancer must always associate oncologic efficiency with function preservation. Traditionally, initial glottic carcinomas (T1) can be effectively treated through surgery or radiotherapy, with high local control and survival rates ${ }^{4,5}$. Despite radiotherapy's complications such as persistent edema, glottic stenosis or hypothyroidism, its advantage is that phonation and swallowing disorders are usually less impacting that the surgical procedures ${ }^{6,7}$. According to Brasil et al. ${ }^{8,9}$, while radiotherapy tends to keep the glottic region as the sound source, in patients submitted to surgery such source tends to shift to the supraglottic region, with a worsening in vocal quality, but keeping a socially acceptable voice, especially when there is speech therapy associated.

According to Bron et al. ${ }^{3}$, T1a carcinomas had similar local control in 5 years with radiotherapy or cordectomy, $77 \%$ and $84 \%$ respectively. In T1b carcinomas, radiotherapy had lower success rates when compared to surgery (supra-cricoid partial laryngectomy), 66\% and $100 \%$ respectively. Other papers report 80 to $95 \% \%^{10,11}$ success rate of radiotherapy in T1 laryngeal carcinomas. Our results with radiotherapy to treat $\mathrm{T} 1 \mathrm{a}$ and $\mathrm{T} 1 \mathrm{~b}$ tumors showed high rates of recurrence. Therefore, we believe that surgical treatment is the best option to control this disease, especially in T1b cases with anterior commissure invasion suspicion.

There is a current trend towards performing more conservative surgical procedures in cases of tumor recurrence after radiotherapy, instead of doing a total laryngectomy. In a study carried out by Schwaab et al. ${ }^{12}$, patients with initial glottic tumors (T1a, T1b and T2 with preserved vocal cord mobility) who had recurrence after radiotherapy were submitted to total or partial laryngectomy (vertical or subtotal with crico-hyoid-pexia) with $77 \%$ and $100 \%$ loco regional control in 5 years, respectively. According to Ganly et al. ${ }^{13}$, patients with recurrence after radiotherapy for initial tumors, treated by total or partial laryngectomy, had global survival rates of $50 \%$ and $89 \%$, respectively, with similar rates of complications, showing that there can be good disease control and preservation of laryngeal functions with partial surgery, when feasible ${ }^{14}$.

Among partial laryngectomies, the endoscopic approach in cases of post-radiotherapy recurrence must be avoided, having in mind the low rate of loco regional disease control, besides missing on an opportunity to do an open surgery with partial laryngeal preservation, as shown in the present study. This is explained by the difficulty in establishing lesion margins in an accurately way in a previously irradiated larynx, associated with the limitation of achieving broad margins.

\section{CONCLUSION}

In our cases, initial glottic tumors (T1) treated by radiotherapy had high rates of recurrence. This can be a treatment option, but the patient must be aware that higher cure rates can be achieved through surgery.

Open partial laryngectomy without neck dissection is an efficient option to treat glottic cancer after radiotherapy failure in patients who remain in stages $\mathrm{T} 1 \mathrm{a}$ or $\mathrm{T} 1 \mathrm{~b}$, bearing satisfactory cancer control without the mutilation of a total laryngectomy. 


\section{REFERENCES}

1. Sjögren EV. Epidemiology of head and neck squamous cell carcinoma. In: Baatenburg de Jong RJ, editors Prognosis in head and neck cancer.London: Taylor and Francis; 2006.p.37-70.

2. Davis RK, Kelly SM, Parkin JL, Stevens MH, Johnson LP. Selective management of early glottic cancer. Laryngoscope. 1990 100(12):1306-9.

3. Bron LP, Soldati D, Zouhair A, Ozsahin M, Brossard E, Monnier P, et al. Treatment of early stage squamous-cell carcinoma of the glottic larynx: endoscopic surgery or cricohyoidoepiglottopexy versus radiotherapy. Head Neck. 2001;23:823-9.

4. Harwood AR, Hawkins NV, Keane T, Cummings B, Beale FA, Rider WD, et al.Radiotherapy of early glottic cancer. Laryngoscope.1980;90:465-70.

5. Smitt MC, Goffinet DR. Radiotherapy for carcinoma-in-situ of the glottic larynx. Int J Radiat Oncol Biol Phys.1993;28:251-5.

6. Nguyen C, Naghibzadeh B, Black MJ, Rochon L, Shenouda G. Carcinoma in situ of the glottic larynx: excision or irradiation? Head Neck. 1996;18:225-8.

7. Sjögren EV, Wiggenraad RGJ, Cessie SL, Snijder S, Pomp J, Jong RJB. Outcome of radiotherapy in T1 glottic carcinoma: a population-based study. Eur Arch Otorhinolaryngol. 2009;266:735-44.
8. Brasil OC, Pontes P, Behlau M. Vertical parcial laryngectomies: oncological control and vocal quality. Phonoscope. 1998;1:283.

9. Brasil OC, Behlau M. Laringectomias parciais verticais: avaliação funcional. Rev Bras Otorrinolaringol. 1999;65:65-76.

10. Nishimura Y, Nagata Y, Okajima K, Mitsumori M, Hiraoka M, Masunaga S, et al. Radiation therapy for T1, 2 glottic carcinoma: impact of overall treatment time on local control. Radiother Oncol. 1996;40:22532.

11. Terhaard CH, Snippe K, Ravasz LA, van der Tweel I, Hordijk GJ. Radiotherapy in T1 laryngeal cancer: prognostic factors for locoregional control and survival, uni- and multivariate analysis. Int J Radiat Oncol Biol Phys. 1991;21:1179-86.

12. Schwaab G, Mamelle G, Lartigau E, Parise O Jr, Wibault P, Luboinski B. . Surgical salvage treat-ment of T1/T2 glottic carcinoma after failure of radiotherapy. Am J Surg. 1994;168(5):474-5.

13. Ganly I, Patel SG, Matsuo J, Singh B, Kraus DH, Boyle JO, et al. Results of surgical salvage after failure of definitive radiation therapy for early-stage squamous cell carcinoma of the glottic larynx. Arch Otolaryngol Head Neck Surg. 2006;132:59-66.

14. Shah JP, Loree TR, Kowalski L. Conservation surgery for radiation failure carcinoma of the glottic larynx. Head Neck. 1990;12:326-31. 